\title{
THE LONGEST CHAIN AMONG RANDOM POINTS IN EUCLIDEAN SPACE
}

\author{
BÉLA BOLLOBÁS ${ }^{1}$ AND PETER WINKLER ${ }^{2}$ \\ (Communicated by Thomas H. Brylawski)
}

\begin{abstract}
Let $n$ random points be chosen independently from the uniform distribution on the unit $k$-cube $[0,1]^{k}$. Order the points coordinate-wise and let $\mathbf{H}_{k}(n)$ be the cardinality of the largest chain in the resulting partially ordered set.

We show that there are constants $c_{1}, c_{2}, \ldots$ such that $c_{k}<e, \lim _{k \rightarrow \infty} c_{k}=$ $e$, and $\lim _{n \rightarrow \infty} \mathbf{H}_{k}(n) / n^{1 / k}=c_{k}$ in probability. This generalizes results of Hammersley, Kingman and others on Ulam's ascending subsequence problem, and settles a conjecture of Steele.
\end{abstract}

1. Introduction. Let $k$ and $n$ be fixed, and let $n$ random points $\vec{x}(1), \vec{x}(2), \ldots$, $\vec{x}(n)$ be chosen independently from the uniform distribution on the unit cube $[0,1]^{k}$ in Euclidean $k$-space. The points then form the underlying set of a random order $\mathbf{P}_{k}(n)$, with partial ordering given by $\vec{x}(i) \leq \vec{x}(j)$ just when $x_{m}(i) \leq x_{m}(j)$ for each $m, 1 \leq m \leq k$. These random orders were introduced as an object of study in [14 and 15] but had certainly appeared many times before in various guises, some of which shall be mentioned below.

Let $\mathbf{H}_{k}(n)$ be the height of $\mathbf{P}_{k}(n)$, i.e. the number of elements in a longest chain (totally ordered subset) of $\mathbf{P}_{k}(n)$. We are interested in the asymptotic behavior of $\mathbf{H}_{k}(n)$ for fixed $k$ and large $n$.

This problem-especially for $k=2$-has quite a colorful history. Note that there is an equivalent "discrete" construction of $\mathbf{P}_{k}(n)$ : fix an underlying set $S$ of $n$ elements, let $<_{1},<_{2}, \ldots,<_{k}$ be random linear orderings of $S$, and put $x<y$ in $\mathbf{P}_{k}(n)$ when $x<_{i} y$ for every $i$. For the purpose of computing $\mathbf{H}_{k}(n)$, we may assume $S=\{1,2, \ldots, n\}$ and $<_{1}$ is the natural ordering of the integers; each subsequent $<_{i}$ is determined by a random permutation $\sigma_{i}$ of $S$ via $x<_{i} y$ iff $\sigma_{i}^{-1}(x)<_{1} \sigma_{i}^{-1}(y)$. It follows that $\mathbf{H}_{2}(n)$ in particular is the length of the longest increasing subsequence of $\sigma_{2}$.

The question of the length of the longest increasing subsequence of a random permutation was apparently first raised by Ulam [12], and since then has often been called "Ulam's problem." Ulam was inspired by the 1935 result of Erdös and Szekeres [2] to the effect that every permutation of $\{1,2, \ldots, n\}$ has either an increasing or decreasing subsequence of length at least $\sqrt{n}$. Monte Carlo computations led Ulam to suspect that $\mathbf{H}_{2}(n)$ tended to some constant multiple of $\sqrt{n}$.

Some years later more extensive computations by Baer and Brock [1] led them to conjecture the value 2 for this constant, but it was Hammersley who lent theoretical

Received by the editors March 19, 1987.

1980 Mathematics Subject Classification (1985 Revision). Primary 06A10, 60D05.

${ }^{1}$ Research supported by MCS grant 8104854 .

${ }^{2}$ Research supported by NRO grant NR539016/5-17-85410.

(C) 1988 American Mathematical Society $0002-9939 / 88 \$ 1.00+\$ .25$ per page 
weight to the conjecture and proved that $\mathbf{H}_{2}(n)$ does tend, at least in probability, to $c \sqrt{n}$ for some constant $c$. In his well-known paper [3], Hammersley made ingenious use of the property of subadditivity to establish the existence of $c$. He went on to prove $\pi / 2 \leq c \leq e$ and to give heuristic arguments for the value $c=2$.

Subsequently Kingman [6] narrowed the gap to $\sqrt{8 / \pi} \leq c<2.49$, and in a comment to Kingman's paper, Kesten [5] showed that if for different $n$ the random permutations were defined on the same probability space in such a way that $\mathrm{H}_{2}(n)$ is nondecreasing, then $\lim _{n \rightarrow \infty} \mathbf{H}_{2}(n) / \sqrt{n}=c$ with probability 1 . (This then holds, in particular, when each successive $\mathbf{P}_{2}(n)$ is obtained by adding a new random point to the unit square.)

In 1977 Logan and Shepp [7] used difficult variational methods to prove that $c \geq$ 2. Their work made use of a result of Schensted [9] tying monotonic subsequences of permutations to shapes of Young tableaux. Finally, Veršik and Kerov [13] obtained $c \leq 2$, settling the conjecture; a combinatorial proof of this result has recently been supplied by Pilpel [9].

Since the interpretation of $\mathbf{H}_{2}(n)$ in terms of random points in a square was used already by Hammersley in [3], it is perhaps surprising that the multi-dimensional analogue to Ulam's problem was apparently not considered until 1977, when Steele [11] used it to attack a statistical problem of Robertson and Wright. Steele conjectured that a constant $c_{k}$ corresponding to Hammersley's " $c$ " exists in every dimension $k$, in particular that $\mathbf{H}_{k}(n) / n^{2^{1-k}} \rightarrow c_{k}$ for some $c_{k}>0$. Although analogous constants do exist it should be noted that there is a discrepancy in exponent between the results below and those in $[\mathbf{1 1}]$, for $k>2$. We believe the difficulty lies at the top of p. 398 of [11], where equation (1.4) appears to require that every chain in a partial order lies inside one of the chains of a fixed Dilworth decomposition.

In what follows we use methods analogous to those of Hammersley and Kingman to obtain our main result.

THEOREM. There are constants $c_{1}, c_{2}, \ldots$ such that

(1) each $c_{k}<e$,

(2) $\lim _{k \rightarrow \infty} c_{k}=e$, and

(3) for each $k, \mathbf{H}_{k}(n) / n^{1 / k}$ tends to $c_{k}$ in probability as $n \rightarrow \infty$.

PROOF. We show first that for each fixed $k$, a constant $c_{k}$ exists with property (3) above. This can be done by applying some very powerful results of subadditive ergodic theory (such results can be used to get a stronger limit statement - see Remark 1 below) but we believe that in this case it is more enlightening to proceed by elementary methods. Throughout the proof, we assume when convenient that large real numbers are integers.

Define $c_{k}=\limsup E\left(\mathbf{H}_{k}(n) / n^{1 / k}\right)$; we begin by showing that $c_{k}<\infty$. Note first that for any $m$, the probability that $m$ given points from $\mathbf{P}_{k}(n)$ form a chain is exactly $1 /(m !)^{k-1}$; for, in the discrete construction of $\mathbf{P}_{k}(n)$, the restriction of the first linear ordering to the $m$ points can be arbitrary but the orders induced by the remaining $k-1$ linear orderings must then be identical to the first. It follows that if $\mathbf{X}_{k}(m, n)$ is the number of chains of length $m$ in $\mathbf{P}_{k}(n)$, then

$$
E\left(\mathbf{X}_{k}(m, n)\right)=\left(\begin{array}{c}
n \\
m
\end{array}\right) /(m !)^{k-1}
$$


Fix any number $c>e$ and let $m=c n^{1 / k}$. Then we have that

$$
E\left(\mathbf{X}_{k}(m, n)\right)=m !\left(c n^{1 / k} !\right)^{-k} /\left(n-c n^{1 / k}\right) !
$$

which, after application of Stirling's formula, turns out to be bounded above by $(e / c)^{c k n^{1 / k}}$. Now,

$$
\begin{aligned}
E\left(\mathbf{H}_{k}(n) / n^{1 / k}\right) & \leq n^{-1 / k}\left[c n^{1 / k} P\left(\mathbf{H}_{k}(n) \leq c n^{1 / k}\right)+n P\left(\mathbf{H}_{k}(n)>c n^{1 / k}\right)\right] \\
& \leq c+n^{1-1 / k}(e / c)^{c k n^{1 / k}} \rightarrow c \quad \text { as } n \rightarrow \infty .
\end{aligned}
$$

It follows that $c_{k} \leq e$. The strict inequality of (1) requires a more subtle argument which we postpone until after showing that $c_{k}$ is actually the limit, in probability, of $\mathbf{H}_{k}(n) / n^{1 / k}$.

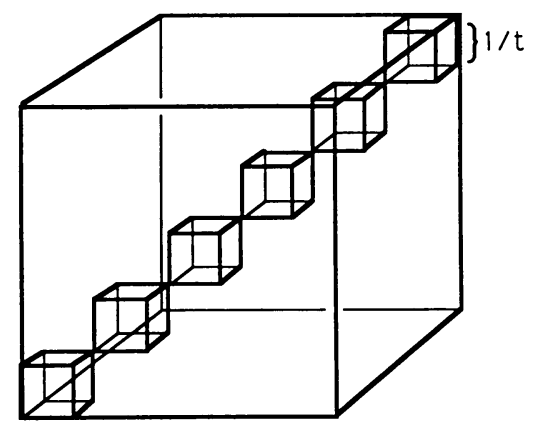

FIGURE 1. Diagonal subcubes of side $1 / t$

Fix $\varepsilon$ with $0<\varepsilon<1 / 10$. for any positive integer $t$ let $D_{1}, \ldots, D_{t}$ be the diagonal subcubes of the unit $k$-cube, given by $D_{i}=[(i-1) / t, i / t]^{k}$. (See Figure 1.) If $n=$ $s t^{k}$ and $\mathbf{Z}_{i}$ is the number of points of $\mathbf{H}_{k}(n)$ in the subcube $D_{i}$, then $\mathbf{Z}_{i}$ is binomially distributed with mean $n\left(1 / t^{k}\right)=s$ and standard deviation $\sqrt{n\left(1 / t^{k}\right)\left(t^{k}-1\right) / t^{k}} \leq$ $\sqrt{s}$. It follows that sufficiently large $s$ will ensure that $P\left(\mathbf{Z}_{i}<s(1-\varepsilon)\right)$ is less than (say) $\varepsilon / 2$ for all $t$. Since the $\mathbf{Z}_{i}$ 's are negatively correlated for different values of $i$, the expected number of "bad" cubes $D_{i}$ containing fewer than $s(1-\varepsilon)$ points of $\mathbf{H}_{k}(n)$ would then be less than $t(\varepsilon / 2)$; and finally, for $s$ greater than some $s_{0}$; the probability that the number of bad cubes exceeds $t \varepsilon$ will be less than $\varepsilon$.

Now let us choose $s_{1}$ such that $\mathbf{H}_{k}\left(s_{1}\right) / s_{1}^{1 / k}>c_{k}-\varepsilon$, and $s_{1}>s_{0}$. Using the law of large numbers, choose $t_{1}$ large enough so that $t>t_{1}$ implies that with probability at least $1-\varepsilon$, the sum of $t(1-\varepsilon)$ independent copies of $\mathbf{H}_{k}\left(s_{1}\right)$ is greater than $t(1-\varepsilon) s_{1}^{1 / k}\left(c_{k}-2 \varepsilon\right)$.

Set $n_{1}=t_{1}^{k} s_{1} /(1-\varepsilon)$ and let $n>n_{1}$, say $n=t^{k} s_{1} /(1-\varepsilon)$. Then with probability at least $1-\varepsilon$, at least $t(1-\varepsilon)$ of the diagonal subcubes of side $1 / t$ contain at least $s_{1}$ points each. In that case let $Y_{i}$ be the length of the longest chain among the first $s_{1}$ points in a "good" cube $D_{i}$; the sum of the $Y_{i}$ 's will then be greater than $t(1-\varepsilon) s_{1}^{1 / k}\left(c_{k}-2 \varepsilon\right)$ with probability at least $1-\varepsilon$.

Since chains in the diagonal subcubes combine to form chains in $\mathbf{P}_{k}(n)$, we have that, with probability at least $1-2 \varepsilon$,

$$
\mathbf{H}_{k}(n) / n^{1 / k}>n^{-1 / k} t(1-\varepsilon) s_{1}^{1 / k}\left(c_{k}-2 \varepsilon\right)=(1-\varepsilon)^{1+1 / k}\left(c_{k}-2 \varepsilon\right) .
$$


Since $n_{1}$ can also be taken large enough to ensure that $n>n_{1}$ implies

$$
E\left(\mathbf{H}_{k}(n) / n^{1 / k}\right)<c_{k}+\varepsilon
$$

and since all this holds for arbitrary small $\varepsilon$, the conclusion is that $\mathbf{H}_{k}(n) / n^{1 / k}$ approaches $c_{k}$ in probability and statement (3) is thus established.

Next we show that the constants $c_{k}$ are bounded strictly by $e$. If $\mathbf{X}_{k}(m, n)$ is the number of $m$-chains in $\mathbf{P}_{n}(k)$, then we must have

$$
\mathbf{X}_{k}(m, n) \geq\left(\begin{array}{c}
\mathbf{H}_{k}(n) \\
m
\end{array}\right)
$$

since a chain of length $\mathbf{H}_{k}(n)$ contains that many subchains of length $m$. It follows that for arbitrary $r \geq m$,

$$
E\left(\mathbf{X}_{k}(m, n)\right) \geq P\left(\mathbf{H}_{k}(n) \geq r\right)\left(\begin{array}{c}
r \\
m
\end{array}\right) \text {. }
$$

Therefore

$$
\begin{aligned}
P\left(\mathbf{H}_{k}(n) \geq r\right) & \leq\left(\begin{array}{c}
n \\
m
\end{array}\right) /\left(\begin{array}{c}
r \\
m
\end{array}\right) m !^{k-1} \\
& \leq n^{m} /(m !)^{k}\left(\begin{array}{c}
r \\
m
\end{array}\right) \leq n^{m} /\left(\frac{m}{e}\right)^{k m}\left(\begin{array}{c}
r \\
r-m
\end{array}\right) .
\end{aligned}
$$

We now set $m=\alpha n^{1 / k}$ and $r=\beta n^{1 / k}$ with the object of obtaining values for $\alpha$ and $\beta$ such that $\alpha<\beta<e$ and the above expression tends to zero as $n \rightarrow \infty$. We then have that the expression is asymptotically equal to

$$
\begin{gathered}
(e / \alpha)^{k m}((\beta-\alpha) / \beta)^{(\beta-\alpha) n^{1 / k}}(\alpha / \beta)^{\alpha n^{1 / k}}=\left[(e / \alpha)^{k}((\beta-\alpha) / \beta)^{\beta / \alpha-1}(\alpha / \beta)\right]^{\alpha n^{1 / k}} \\
=\left[e^{k} \alpha^{-(k-1)} \beta^{-\beta / \alpha}(\beta-\alpha)^{\beta / \alpha-1}\right]^{\alpha n^{1 / k}}=\left[e^{k \alpha} \alpha^{-(k-1) \alpha} \beta^{-\beta}(\beta-\alpha)^{\beta-\alpha}\right]^{n^{1 / k}}
\end{gathered}
$$

therefore it will suffice to obtain

$$
k \alpha-(k-1) \alpha \log \alpha-\beta \log \beta+(\beta-\alpha) \log (\beta-\alpha) \leq 0 .
$$

Setting $\alpha=e(1-\gamma)$ and $\beta=e(1-\delta)$, the left-hand side of the above inequality becomes equal to

$$
\begin{aligned}
k(1-\gamma)- & (k-1)(1-\gamma)[1+\log (1-\gamma)]-(1-\delta)[1+\log (1-\delta)] \\
& +(\gamma-\delta)[\log (\gamma-\delta)+1] \\
= & -(k-1)(1-\gamma) \log (1-\gamma)-(1-\delta) \log (1-\delta)+(\gamma-\delta) \log (\gamma-\delta) \\
\leq & -(k-1)(1-\gamma)(-\gamma)-(1-\delta)(-\delta)+(\gamma-\delta) \log (\gamma-\delta) \\
\leq & (k-1) \gamma+\delta+(\gamma-\delta) \log (\gamma-\delta) .
\end{aligned}
$$
and

Finally, set $\gamma=(1+1 / k) e^{-k}$ and $\delta=(1 / k) e^{-k} ;$ then $(\gamma-\delta) \log (\gamma-\delta)=-k e^{-k}$

$$
(k-1)(1+1 / k) e^{-k}+(1 / k) e^{-k}-k e^{-k}=0,
$$

proving part (1) of the Theorem.

To show that the constants $c_{k}$ approach $e$ we must build a chain in $\mathbf{P}_{k}(n)$; this we do from the bottom up. It is convenient, however, to obtain $\mathbf{P}_{k}(n)$ in a somewhat different way. Let $\vec{x}(1), \vec{x}(2), \ldots$ be the points of a Poisson process of density 1 in $\mathbf{R}^{k}$. Then for some real $r$ depending on the outcome of the process, the cube $[0, r]^{k}$ 


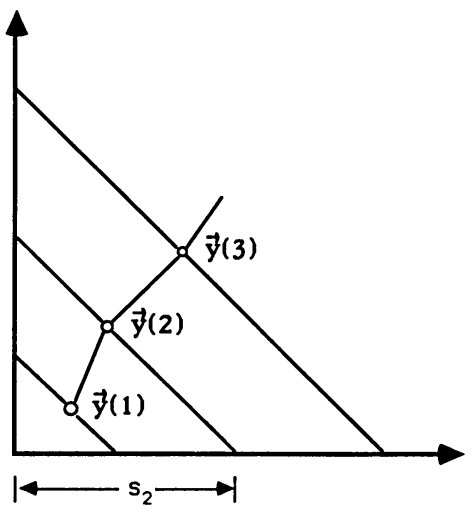

FIgURE 2. Bottom-up chain construction in a Poisson process

will contain exactly $n$ points of the process and will yield a model of $\mathbf{P}_{\boldsymbol{k}}(n)$ by the usual partial ordering. Moreover, fixing $\varepsilon>0$, we can choose $n_{2}$ such that $n>n_{2}$ implies that with probability at least $1-\varepsilon, r$ will be at least $(1-\varepsilon) n^{1 / k}$.

We now construct a chain among the points of the process as follows. Choose $\vec{y}(1)=\left(y_{1}(1), y_{2}(1), \ldots, y_{k}(1)\right)$ from the $\vec{x}(j)$ 's such that each $y_{i}(1)>0$ and $s_{1}=$ $y_{1}(1)+\cdots+y_{k}(1)$ is minimal. Thereafter, $\vec{y}(j)$ is the point satisfying $\vec{y}(j)>\vec{y}(j-1)$ for which $s_{j}$ is minimal. (See Figure 2.)

Since the region $\left\{\vec{x}: \vec{x}>\overrightarrow{0}\right.$ and $\left.\sum_{i=0}^{k} x_{i} \leq s\right\}$ is with probability $e^{-s^{k} / k !}$ unoccupied by a point of the Poisson process, the mass density of the random variable $s_{1}$ is given by the function

$$
f(s)=\frac{d}{d s}\left(1-e^{-s^{k} / k !}\right)=\frac{s^{k-1}}{(k-1) !} e^{-s^{k} / k !}
$$

and thus the expected value of $s_{1}$ is

$$
\begin{aligned}
\int_{0}^{\infty} \frac{s^{k}}{(k-1) !} e^{-s^{k} / k !} d s & =\int_{0}^{\infty} \frac{k ! t}{(k-1) !} e^{-t} \frac{1}{k}(k ! t)^{1 / k-1} k ! d t \\
& =k !^{1 / k} \Gamma(1+1 / k) .
\end{aligned}
$$

Now for each $i$, the differences $x_{i}(j)-x_{i}(j-1)$ and $x_{i}(1)-0$ are independent and identically distributed with mean

$$
E\left(s_{1}\right) / k=k^{-1} k !^{1 / k} \Gamma(1+1 / k)=k^{-2} k !^{1 / k} \Gamma(1 / k) .
$$

It follows from the law of large numbers that for sufficiently large $m, Y_{i}(m)<$ $(1+\varepsilon) m\left(k^{-2} k !^{1 / k}\right) \Gamma(1 / k)$ for every $i$, with probability at least $1-\varepsilon$. Choosing

$$
m=\frac{(1-\varepsilon) n^{k} k^{2}}{(1+\varepsilon) k !^{1 / k} \Gamma(1 / k)}
$$

for $n$ greater than some $n_{3}$, we then have that with probability at least $1-\varepsilon$, the point $\vec{Y}(m)$-and therefore a chain of length $m$-lies in the cube $\left[0,(1-\varepsilon) n^{1 / k}\right]^{k}$. When $n$ is also greater than $n_{2}$ this chain lies, with probability at least $1-2 \varepsilon$, within $\mathbf{P}_{k}(n)$. Letting $\varepsilon$ go to zero we thus have that

$$
c_{k} \geq k^{2} / k !^{1 / k} \Gamma(1 / k) \text {. }
$$


This agrees with the result that $c_{2} \geq \sqrt{8 / \pi}$ obtained by Kingman [6] using the above method in the plane.

It remains only to note that

$$
\lim _{k \rightarrow \infty} \frac{k^{2}}{k !^{1 / k} \Gamma(1 / k)}=\lim _{k \rightarrow \infty} \frac{k^{2}}{\left((k / e)^{k}\right)^{1 / k} k}=e
$$

so that $c_{k} \rightarrow e$ and the proof of the Theorem is complete.

REMARK 1. It is natural to ask whether, when the random variables $\mathbf{H}_{k}(n)$ are defined on a common sample space, the stronger statement that

$$
\lim _{n \rightarrow \infty} \mathbf{H}_{k}(n) / n^{1 / k}=c_{k}
$$

with probability 1 can be substituted for statement (3) of the Theorem. The most obvious sample space (in our context) is obtained by choosing an infinite sequence of random points in the unit $k$-cube, letting $\mathbf{H}_{k}(n)$ be the height of the $\mathbf{P}_{k}(n)$ determined by the first $n$ points. In that case the $\mathbf{H}_{k}(n)$ 's are of course monotone, and Kesten's comment in [5] applies here exactly as it does in the case $k=2$. In particular the lemma of Note 7 in Hammersley [4], which strengthens Kesten's result, can be applied to obtain the stronger limit statement.

REMARK 2. Several interesting questions remain - for example, what are the values of the constants $c_{k}$ ? The difficulty of establishing the value of $c_{2}$ does not bode well. Moreover, although it is algorithmically very easy to determine the height of a partially ordered set, our own Monte Carlo efforts to determine $c_{3}$ have not yet even suggested a conjecture for that value.

Since the sequence of $c_{k}$ 's begins with $1,2, \ldots$ and approaches $e$ it appears likely that it is monotone increasing, but the upper and lower bounds for $c_{k}$ obtained in the proof of the main theorem above are not close enough to obtain that result.

\section{REFERENCES}

1. R. M. Baer and P. Brock, Natural sorting over permutation spaces, Math. Comp. 22 (1968), 385-510.

2. P. Erdős and Gy. Szekeres, A combinatorial problem in geometry, Compositio Math. 2 (1935), 463-470.

3. J. M. Hammersley, A few seedlings of research, Proc. Sixth Berkeley Sympos. Math. Stat. Prob., Univ. of California Press, Berkeley, Calif., 1972, pp. 345-394.

4. _ Postulates for subadditive processes, Ann. Probab. 2 (1974), 652-680.

5. H. Kesten, Comment on J. F. C. Kingman's article, Ann. Probab. 1 (1973), p. 903.

6. J. F. C. Kingman, Subadditive ergodic theory, Ann. Probab. 1 (1973), 883-909.

7. B. F. Logan and L. A. Shepp, A variational problem for random Young tableaux, Adv. in Math. 26 (1977), 206-222.

8. S. Pilpel, Descending subsequences of random permutations, IBM Research Report \#52283, 1986.

9. C. Schensted, Longest increasing and decreasing subsequences, Canad. J. Math. 13 (1961), 179191.

10. S. M. Samuels and J. M. Steele, Optimal sequential selection of a monotone sequence from a random sample, Ann. Probab. 9 (1981), 937 947.

11. J. M. Steele, Limit properties of random variables associated with a partial ordering of $R^{d}$, Ann. Probab. 5 (1977), 395403.

12. S. M. Ulam, Monte Carlo calculations in problems of mathematical physics, Modern Mathematics for the Engineer (E. F. Beckenbach, Ed.), McGraw-Hill, New York, 1961. 
13. A. M. Versik and S. V. Kerov, Asymptotics of the Plancherel measure of the symmetric group and the limiting form of Young tableaux, Dokl. Akad. Nauk SSSR 233 (1977), 1024-1028.

14. P. Winkler, Random orders, Order 1 (1985), 317-331.

15. _, Connectedness and diameter for random orders of fixed dimension, Order 2 (1985), 165171.

Department of Mathematics, Louisiana State University, Baton Rouge, LOUISIANA 70803

Department of PuRe Mathematics and Mathematical Statistics, Cambridge UNIVERSITY, CAMBRIDGE CB2 1SB, ENGLAND

Department of Mathematics and Computer SCIENCE, EMory UNiversity, Atlanta, Georgia 30322 